\title{
UBV photometry of the asteroid 44 Nysa
}

\author{
F. A. Tupieva ${ }^{\star}$ \\ Institute of Astrophysics, Tajik Academy of Sciences, and Isaac Newton Institute of Chile, Tajikistan Branch, Bukhoro Str. 22, \\ Dushanbe 734042, Tajikistan \\ Received 10 June 2002 / Accepted 30 April 2003

\begin{abstract}
The color curves of Nysa connected to the rotation and phase angle were obtained from observations for the first time in 1982. The color curves showed the $U-B$ change with rotation. The value of this change was 0.2 mag. Color variation indicates that the extended color spot is on the surface of 44 Nysa. The $U-B$ color index decreases with the increase of the phase angle (the phase coefficient is $0.011 \mathrm{mag} / \mathrm{deg}$ ). This decrease is stronger for the surface with color spots. Phase functions of the brightness for opposite sides of Nysa are different. The value of the opposition effect of 44 Nysa decreases as reflectance decreases.
\end{abstract}

Key words. minor planets, asteroids - star system: general - techniques: photometric

\section{Introduction}

44 Nysa belongs to a rare type of E-asteroid having high reflectivity (40\%). It is a member of an unusual family that contains the large M-type asteroid 135 Hertha and a large number of F-type asteroids. The investigation of the members of this family is interesting for the study of the homogeneity of their surfaces, because the members of the Nysa family probably have been formed from differentiated parent bodies (Degewij et al. 1979). Nysa also has unusual opposition effects (Harris et al. 1989a): the wideness of the opposition peak is smaller by 3-4 times than that of other asteroids.

Beginning in 1949, when the first electrophotometric measurements of the brightness of Nysa were obtained, this asteroid has been observed photometrically during more then ten oppositions. Individual measurements of $U-B$ and $B-V$ color indices of Nysa are given by Groeneveld \& Kuiper (1954), Zappala \& van Houten-Groeneveld (1979), Piironen (1982), Taylor \& Tedesco (1983). However, data of color variations during a rotational cycle and dependence of color on the phase angle have not been obtained. Phase color curves are useful for the interpretation of the opposition effect (Helfenstein et al. 1997) and color variations with rotation for the definition of Nysa's surface homogeneity.

In this paper, phase color curves and color variation of 44 Nysa, obtained with $U B V$ broadband photometry, are discussed.

\section{Observations}

In July-August 1982 the photoelectric measurements of the brightness of 44 Nysa were carried out with the $70-\mathrm{cm}$

* e-mail: fiza@ac.tajik.net
Table 1. The data of comparison stars.

\begin{tabular}{|c|c|c|c|c|c|}
\hline 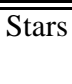 & $\begin{array}{c}\delta(2000.0) \\
\mathrm{h} \mathrm{m}\end{array}$ & $\begin{array}{c}\delta(2000.0) \\
\text { deg min }\end{array}$ & $\begin{array}{c}V \\
\text { mag }\end{array}$ & $\begin{array}{r}U-B \\
\text { mag }\end{array}$ & $\begin{array}{c}\begin{array}{c}B-V \\
\text { mag }\end{array} \\
\end{array}$ \\
\hline$\overline{1}$ & 1924.8 & -1933.0 & 10.00 & -0.03 & 0.50 \\
\hline 2 & 1921.2 & -1945.9 & 10.38 & 0.61 & 1.06 \\
\hline 3 & 1913.6 & -2011.3 & 9.66 & 1.57 & 1.43 \\
\hline 4 & $19 \quad 03.1$ & -2043.0 & 8.51 & 0.12 & 0.56 \\
\hline 5 & $18 \quad 52.5$ & -2119.0 & 9.57 & 0.27 & 0.31 \\
\hline
\end{tabular}

reflector of the Gissar Observatory of Tajikistan at $U B V$ standard spectral bands. Conditions of observation of the asteroid were difficult: the airmass of the asteroid was close to 2, the observations were made at full Moon and there were nights affected by the presence of dust in the Earth's atmosphere. Therefore, comparison stars were selected close to the asteroid position and they were often compared with photometric standards and among themselves. Observations were carried out with the help of a photometer, working in the regime of the count of photons. Counting time of the signal was $30 \mathrm{~s}$. 2 to 4 measurements of the asteroid's brightness were made between two measurements of a star's brightness. The stability of operation of the photometer was controlled by the sample, the brightness of which was measured for $40 \mathrm{~min}$ on average. The accuracy of single measurement of the asteroid's brightness was of 0.015 in the $V$ band, 0.02 in $B-V$ and 0.04 in $U-B$.

The data of comparison stars are given in Table 1.

Their coordinates were determined by the Stellar atlas of Vehrenberg (1970). Their brightness and color indices were determined by comparison with photometric standards IC 4275 (№ 115, № 123) and SA112 (№ 1242, № 1333, № 1370) (Kazanasmas et al. 1981; Moffett \& Barnes 1979). 
Table 2. The aspect data for Nysa in 1982.

\begin{tabular}{|c|c|c|c|c|c|c|c|}
\hline Date, UT & $\begin{array}{c}\alpha(2000.0) \\
\mathrm{h} \mathrm{m}\end{array}$ & $\begin{array}{c}\delta(2000.0) \\
\text { deg min }\end{array}$ & $\begin{array}{c}\lambda(2000.0) \\
\text { deg }\end{array}$ & $\begin{array}{c}\beta(2000.0) \\
\operatorname{deg}\end{array}$ & $\begin{array}{c}r \\
\mathrm{au}\end{array}$ & $\begin{array}{l}\Delta \\
\mathrm{au}\end{array}$ & $\begin{array}{r}\alpha \\
\operatorname{deg} \\
\end{array}$ \\
\hline $82 / 07 / 03.7758$ & 1926.4 & -1931 & 290.3 & 2.4 & 2.786 & 1.777 & 3.1 \\
\hline $82 / 07 / 08.8154$ & 1921.5 & -1946 & 289.1 & 2.3 & 2.787 & 1.772 & 1.2 \\
\hline $82 / 07 / 09.8221$ & 1920.5 & -1948 & 288.9 & 2.3 & 2.787 & 1.771 & 0.9 \\
\hline $82 / 07 / 11.7962$ & 1918.5 & -1954 & 289.4 & 2.3 & 2.788 & 1.773 & 1.3 \\
\hline $82 / 07 / 14.8417$ & 1915.5 & -2003 & 287.7 & 2.2 & 2.788 & 1.775 & 2.2 \\
\hline $82 / 07 / 16.7849$ & 1913.6 & -2009 & 287.3 & 2.2 & 2.788 & 1.777 & 2.6 \\
\hline $82 / 07 / 18.8209$ & 1911.5 & -2015 & 286.8 & 2.2 & 2.789 & 1.781 & 3.5 \\
\hline $82 / 07 / 24.8186$ & 1906.0 & -2033 & 285.6 & 2.0 & 2.789 & 1.797 & 5.8 \\
\hline $82 / 07 / 26.7699$ & 1904.4 & -2038 & 285.0 & 2.0 & 2.790 & 1.807 & 6.6 \\
\hline $82 / 07 / 28.7742$ & 1902.6 & -2043 & 284.6 & 1.9 & 2.790 & 1.817 & 7.5 \\
\hline $82 / 07 / 30.7585$ & 1900.9 & -2050 & 284.2 & 1.9 & 2.790 & 1.828 & 8.3 \\
\hline $82 / 08 / 08.7340$ & 1854.4 & -2110 & 282.7 & 1.7 & 2.790 & 1.887 & 11.6 \\
\hline $82 / 08 / 09.6730$ & 1853.9 & -2114 & 282.5 & 1.6 & 2.790 & 1.893 & 11.8 \\
\hline $82 / 08 / 14.7212$ & 1851.5 & -2120 & 282.0 & 1.6 & 2.789 & 1.934 & 13.4 \\
\hline $82 / 08 / 22.7299$ & 1848.7 & -2142 & 281.3 & 1.3 & 2.788 & 2.010 & 15.6 \\
\hline
\end{tabular}

Table 3. Brightness and colors of 44 Nysa in 1982.

\begin{tabular}{|c|c|c|c|c|}
\hline $\begin{array}{c}\text { Date, UT } \\
\text { h m }\end{array}$ & $\begin{array}{c}U-B \\
\text { mag }\end{array}$ & $\begin{array}{c}\begin{array}{c}B-V \\
\text { mag }\end{array} \\
\text { ag }\end{array}$ & $\begin{array}{c}V \\
\text { mag }\end{array}$ & 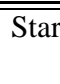 \\
\hline$\overline{3.07}$ & & & & 1 \\
\hline 183712 & 0.23 & 0.75 & 10.57 & \\
\hline 4326 & 0.21 & 0.79 & 10.61 & \\
\hline 8.07 & & & & 2 \\
\hline 181845 & 0.41 & 0.75 & 10.70 & \\
\hline 2708 & 0.37 & 0.72 & 10.71 & \\
\hline 3810 & 0.24 & 0.77 & 10.70 & \\
\hline 4345 & 0.24 & 0.77 & 10.72 & \\
\hline 190511 & 0.22 & 0.79 & 10.71 & \\
\hline 1719 & - & 0.88 & 10.60 & \\
\hline 2229 & 0.36 & 0.80 & 10.56 & \\
\hline 3412 & 0.34 & 0.70 & 10.58 & \\
\hline 3925 & 0.22 & 0.72 & 10.58 & \\
\hline 5018 & 0.25 & 0.70 & 10.53 & \\
\hline 5543 & 0.07 & 0.75 & 10.54 & \\
\hline 200650 & 0.03 & 0.76 & 10.47 & \\
\hline 1231 & 0.10 & 0.78 & 10.52 & \\
\hline 2311 & 0.28 & 0.72 & 10.49 & \\
\hline 2821 & 0.28 & 0.69 & 10.52 & \\
\hline 9.07 & & & & 2 \\
\hline 184538 & 0.37 & 0.78 & 10.54 & \\
\hline 4831 & 0.43 & 0.76 & 10.55 & \\
\hline 5350 & 0.49 & 0.76 & 10.58 & \\
\hline 5618 & 0.57 & 0.76 & 10.60 & \\
\hline 191543 & 0.30 & 0.74 & 10.59 & \\
\hline
\end{tabular}

\begin{tabular}{|c|c|c|c|c|}
\hline $\begin{array}{c}\text { Date, UT } \\
\text { h m } \quad \text { s }\end{array}$ & $\begin{array}{c}U-B \\
\text { mag }\end{array}$ & $\begin{array}{c}\overline{B-V} \\
\text { mag }\end{array}$ & $\begin{array}{c}V \\
\mathrm{mag}\end{array}$ & $\overline{\overline{\text { Star }}}$ \\
\hline 9.07 & & & & 2 \\
\hline 191806 & 0.45 & 0.73 & 10.57 & \\
\hline 2323 & 0.27 & 0.75 & 10.60 & \\
\hline 2551 & 0.33 & 0.72 & 10.62 & \\
\hline 4348 & 0.39 & 0.70 & 10.66 & \\
\hline 4620 & 0.52 & 0.76 & 10.65 & \\
\hline 5136 & 0.39 & 0.78 & 10.66 & \\
\hline 5407 & 0.25 & 0.74 & 10.65 & \\
\hline 201251 & 0.17 & 0.73 & 10.65 & \\
\hline 1518 & 0.30 & 0.70 & 10.65 & \\
\hline 2042 & 0.35 & 0.72 & 10.65 & \\
\hline 2314 & 0.39 & 0.73 & 10.63 & \\
\hline 11.07 & & & & 2 \\
\hline 183517 & 0.35 & 0.80 & 10.50 & \\
\hline 3738 & 0.30 & 0.73 & 10.53 & \\
\hline 4257 & 0.33 & 0.77 & 10.50 & \\
\hline 4527 & 0.39 & 0.74 & 10.51 & \\
\hline 190612 & 0.33 & 0.77 & 10.53 & \\
\hline 0835 & 0.37 & 0.76 & 10.53 & \\
\hline 1342 & 0.39 & 0.78 & 10.55 & \\
\hline 1604 & 0.33 & 0.76 & 10.55 & \\
\hline 14.07 & & & & 3 \\
\hline 195203 & 0.30 & 0.73 & 10.62 & \\
\hline 5439 & 0.26 & 0.77 & 10.59 & \\
\hline 200152 & 0.27 & 0.75 & 10.58 & \\
\hline
\end{tabular}

\begin{tabular}{|c|c|c|c|c|}
\hline $\begin{array}{c}\text { Date, UT } \\
\text { h m s }\end{array}$ & $\begin{array}{c}U-B \\
\text { mag }\end{array}$ & $\begin{array}{r}\begin{array}{c}B-V \\
\text { mag }\end{array} \\
\text { a }\end{array}$ & $\begin{array}{c}V \\
\text { mag }\end{array}$ & $\overline{\text { Star }}$ \\
\hline$\overline{14.07}$ & & & & 3 \\
\hline 201202 & 0.27 & 0.76 & 10.57 & \\
\hline 1734 & 0.32 & 0.73 & 10.57 & \\
\hline 2013 & 0.33 & 0.72 & 10.57 & \\
\hline 2524 & 0.32 & 0.72 & 10.54 & \\
\hline 16.07 & & & & 3 \\
\hline 180130 & - & 0.73 & 10.61 & \\
\hline 0400 & - & 0.73 & 10.62 & \\
\hline 1002 & 0.23 & 0.82 & 10.64 & \\
\hline 1528 & 0.17 & 0.77 & 10.63 & \\
\hline 2549 & 0.23 & 0.77 & 10.64 & \\
\hline 2816 & - & 0.74 & 10.69 & \\
\hline 3425 & 0.24 & 0.74 & 10.70 & \\
\hline 3933 & 0.28 & 0.76 & 10.70 & \\
\hline 5018 & 0.27 & 0.78 & 10.73 & \\
\hline 5253 & 0.18 & 0.78 & 10.75 & \\
\hline 5803 & - & 0.77 & 10.77 & \\
\hline 190314 & 0.19 & 0.77 & 10.71 & \\
\hline 1232 & 0.25 & 0.75 & 10.69 & \\
\hline 1454 & 0.24 & 0.77 & 10.70 & \\
\hline 2057 & 0.31 & 0.73 & 10.70 & \\
\hline 2627 & 0.27 & 0.76 & 10.71 & \\
\hline 18.07 & & & & 3 \\
\hline 191417 & 0.23 & 0.75 & 10.80 & \\
\hline 2001 & 0.18 & 0.77 & 10.80 & \\
\hline
\end{tabular}

Table 2 summarises the aspect data for Nysa in the opposition of 1982. The date and universal time of the middle observation (UT) corrected for light-time are given in the first column. The equatorial $(\alpha, \delta)$ and ecliptic $(\lambda, \beta)$ coordinates of the asteroid at 2000.0, the heliocentric $(r)$ and geocentric $(\Delta)$ distances and the solar phase angle $\left(\alpha^{\circ}\right)$ are given in the following columns.

Table 3 lists the $V$ magnitudes and color indices $(B-V$ and $U-B$ ) derived for Nysa with the standard $U B V$ system for the individual measurements of brightness of the asteroid. The first column lists universal time corrected for light time. The final column lists the comparison stars used (see Table 1).

\section{Lightcurve and color}

In 1982, the conditions of visibility of 44 Nysa did not allow us to observe the asteroid for more than 3 hours during the night, and its sinodic rotation period is 6. 422 (Birch et al. 1983; 
Table 3. continued.

\begin{tabular}{|c|c|c|c|c|}
\hline $\begin{array}{c}\text { Date, UT } \\
\mathrm{h} \quad \mathrm{m} \quad \mathrm{s}\end{array}$ & $\begin{array}{c}U-B \\
\text { mag }\end{array}$ & $\begin{array}{c}B-V \\
\text { mag }\end{array}$ & $\begin{array}{c}V \\
\text { mag }\end{array}$ & Star \\
\hline 18.07 & & & & 3 \\
\hline 192226 & 0.30 & 0.74 & 10.78 & \\
\hline 2823 & 0.21 & 0.76 & 10.79 & \\
\hline 4203 & 0.23 & 0.76 & 10.77 & \\
\hline 4703 & 0.18 & 0.77 & 10.74 & \\
\hline 4915 & 0.19 & 0.73 & 10.75 & \\
\hline 5450 & 0.18 & 0.72 & 10.73 & \\
\hline 24.07 & & & & 4 \\
\hline 191515 & - & 0.73 & 10.83 & \\
\hline 2040 & - & 0.76 & 10.83 & \\
\hline 2255 & 0.29 & 0.69 & 10.85 & \\
\hline 3849 & 0.32 & 0.70 & 10.85 & \\
\hline 4329 & 0.28 & 0.70 & 10.85 & \\
\hline 5110 & 0.22 & 0.69 & 10.81 & \\
\hline 200909 & 0.29 & 0.72 & 10.82 & \\
\hline 26.07 & & & & 4 \\
\hline 170145 & - & 0.70 & 10.88 & \\
\hline 0702 & 0.39 & 0.70 & 10.91 & \\
\hline 0931 & 0.27 & 0.75 & 10.87 & \\
\hline 2345 & 0.24 & 0.76 & 10.85 & \\
\hline 3111 & 0.29 & 0.73 & 10.81 & \\
\hline 3553 & 0.31 & 0.71 & 10.81 & \\
\hline 5508 & 0.32 & 0.73 & 10.70 & \\
\hline 180041 & 0.33 & 0.72 & 10.71 & \\
\hline 0302 & 0.24 & 0.74 & 10.67 & \\
\hline 0759 & 0.30 & 0.73 & 10.69 & \\
\hline 2003 & 0.31 & 0.73 & 10.68 & \\
\hline 2620 & 0.35 & 0.75 & 10.66 & \\
\hline 2846 & 0.27 & 0.74 & 10.68 & \\
\hline 3413 & 0.27 & 0.72 & 10.69 & \\
\hline 4305 & 0.30 & 0.75 & 10.69 & \\
\hline 5028 & 0.35 & 0.74 & 10.67 & \\
\hline 5248 & 0.30 & 0.73 & 10.69 & \\
\hline 5801 & 0.33 & 0.73 & 10.72 & \\
\hline 190805 & 0.26 & 0.74 & 10.75 & \\
\hline 1257 & 0.32 & 0.73 & 10.78 & \\
\hline 1515 & 0.36 & 0.78 & 10.75 & \\
\hline 2013 & 0.29 & 0.74 & 10.77 & \\
\hline 28.07 & & & & 4 \\
\hline 173243 & - & 0.70 & 10.89 & \\
\hline 3806 & 0.26 & 0.72 & 10.86 & \\
\hline
\end{tabular}

\begin{tabular}{|c|c|c|c|c|}
\hline $\begin{array}{c}\text { Date, UT } \\
\mathrm{h} \quad \mathrm{m} \quad \mathrm{s}\end{array}$ & $\begin{array}{c}U-B \\
\text { mag }\end{array}$ & $\begin{array}{c}B-V \\
\text { mag }\end{array}$ & $\begin{array}{c}V \\
\mathrm{mag}\end{array}$ & $\overline{\text { Star }}$ \\
\hline 28.07 & & & & 4 \\
\hline 174103 & 0.23 & 0.72 & 10.85 & \\
\hline 4808 & - & 0.75 & 10.83 & \\
\hline 5721 & - & 0.75 & 10.83 & \\
\hline 180816 & 0.26 & 0.75 & 10.85 & \\
\hline 1035 & 0.24 & 0.73 & 10.84 & \\
\hline 1726 & 0.38 & 0.77 & 10.82 & \\
\hline 2639 & 0.33 & 0.70 & 10.77 & \\
\hline 3231 & 0.22 & 0.68 & 10.78 & \\
\hline 3449 & 0.28 & 0.78 & 10.74 & \\
\hline 4058 & 0.40 & 0.80 & 10.75 & \\
\hline 5334 & 0.42 & 0.68 & 10.77 & \\
\hline 5849 & 0.31 & 0.78 & 10.73 & \\
\hline 190159 & 0.38 & 0.73 & 10.76 & \\
\hline 0753 & 0.39 & 0.78 & 10.76 & \\
\hline 1758 & 0.36 & 0.71 & 10.80 & \\
\hline 2312 & 0.35 & 0.75 & 10.83 & \\
\hline 2539 & - & 0.74 & 10.86 & \\
\hline 3104 & 0.38 & 0.68 & 10.85 & \\
\hline 30.07 & & & & 4 \\
\hline 173720 & - & 0.78 & 10.91 & \\
\hline 3946 & 0.26 & 0.77 & 10.91 & \\
\hline 4525 & 0.25 & 0.75 & 10.91 & \\
\hline 4756 & 0.30 & 0.75 & 10.89 & \\
\hline 180257 & 0.38 & 0.76 & 10.80 & \\
\hline 0506 & 0.34 & 0.71 & 10.84 & \\
\hline 1001 & 0.22 & 0.74 & 10.81 & \\
\hline 1220 & 0.21 & 0.78 & 10.82 & \\
\hline 2636 & 0.33 & 0.72 & 10.77 & \\
\hline 2856 & 0.35 & 0.74 & 10.74 & \\
\hline 3407 & 0.30 & 0.75 & 10.73 & \\
\hline 3627 & 0.32 & 0.75 & 10.76 & \\
\hline 5124 & 0.31 & 0.78 & 10.74 & \\
\hline 5339 & 0.31 & 0.75 & 10.75 & \\
\hline 5849 & - & 0.73 & 10.76 & \\
\hline 190105 & - & 0.74 & 10.74 & \\
\hline 8.08 & & & & 5 \\
\hline 172658 & 0.18 & - & 11.02 & \\
\hline 2920 & 0.22 & 0.77 & 10.99 & \\
\hline 3451 & 0.27 & 0.71 & 10.98 & \\
\hline 3713 & 0.17 & 0.72 & 10.98 & \\
\hline
\end{tabular}

\begin{tabular}{|c|c|c|c|c|}
\hline $\begin{array}{l}\text { Date, UT } \\
\text { h m s }\end{array}$ & $\begin{array}{c}U-B \\
\text { mag }\end{array}$ & $\begin{array}{c}B-V \\
\text { mag }\end{array}$ & $\begin{array}{c}V \\
\mathrm{mag}\end{array}$ & $\overline{\text { Star }}$ \\
\hline$\overline{8.08}$ & & & & 5 \\
\hline 175433 & 0.33 & 0.74 & 10.92 & \\
\hline 5655 & 0.35 & 0.79 & 10.89 & \\
\hline 180158 & - & 0.74 & 10.95 & \\
\hline 0424 & 0.41 & 0.77 & 10.95 & \\
\hline 9.08 & & & & 5 \\
\hline 161824 & 0.36 & 0.73 & 10.91 & \\
\hline 2358 & 0.25 & 0.73 & 10.94 & \\
\hline 2623 & 0.16 & 0.78 & 10.92 & \\
\hline 3130 & 0.17 & 0.78 & 10.92 & \\
\hline 14.08 & & & & 5 \\
\hline 162351 & 0.27 & 0.70 & 11.21 & \\
\hline 2800 & 0.21 & 0.73 & 11.19 & \\
\hline 3030 & 0.35 & 0.72 & 11.18 & \\
\hline 3552 & 0.37 & 0.72 & 11.19 & \\
\hline 4810 & 0.15 & 0.76 & 11.19 & \\
\hline 5420 & 0.31 & 0.76 & 11.19 & \\
\hline 5655 & 0.37 & 0.73 & 11.19 & \\
\hline 170216 & 0.33 & 0.74 & 11.21 & \\
\hline 1221 & 0.22 & 0.72 & 11.19 & \\
\hline 1833 & 0.32 & 0.75 & 11.19 & \\
\hline 2027 & 0.33 & 0.76 & 11.15 & \\
\hline 2534 & 0.31 & 0.77 & 11.14 & \\
\hline 3623 & - & 0.77 & 11.11 & \\
\hline 4141 & 0.42 & 0.73 & 11.09 & \\
\hline 5228 & 0.37 & 0.69 & 11.03 & \\
\hline 5805 & 0.48 & 0.68 & 11.04 & \\
\hline 180900 & 0.17 & 0.70 & 11.01 & \\
\hline 1410 & 0.27 & 0.71 & 11.01 & \\
\hline 1637 & 0.41 & 0.72 & 11.00 & \\
\hline 2145 & 0.45 & 0.79 & 10.99 & \\
\hline 22.08 & & & & 5 \\
\hline 171901 & 0.31 & 0.77 & 11.35 & \\
\hline 2348 & 0.13 & 0.74 & 11.35 & \\
\hline 2615 & 0.35 & 0.78 & 11.36 & \\
\hline 3058 & 0.18 & 0.75 & 11.35 & \\
\hline 4647 & - & 0.74 & 11.36 & \\
\hline 5139 & 0.18 & 0.69 & 11.36 & \\
\hline 5356 & 0.19 & 0.88 & 11.32 & \\
\hline 5849 & 0.35 & 0.83 & 11.38 & \\
\hline
\end{tabular}

Di Martino et al. 1987; Harris et al. 1989a). Thus, we show composite lightcurves. In Fig. 1, two composite lightcurves of 44 Nysa are given: upper - for the interval of phase angles from 0.9 to 3.5 , lower - from 5.8 to 8.3 . The upper composite lightcurve was made from seven observation nights relative to comparison stars № 2 and № 3, and the lower composite lightcurve was compiled from four nights relative to star № 4 (see Table 3). The intervals of rotation phase for each night are given in Table 4 . The zero phase of rotation $(f=0)$ corresponds to $19^{\text {h }}(\mathrm{UT})$ on July 18,1982 . At the calculation of the rotating phase, the light-time was taken into consideration, i.e. the time needed for light to travel the distance from the asteroid to the Earth.

The identification of the extrema of the lightcurves in Fig. 1 corresponds to the definition of Birch et al. (1983). M1 is the maximum that precedes the flat-bottom minimum. There is a notch in the right side of this minimum, which clearly appears on the lightcurves in the opposition of 1979. The amplitude of the obtained lightcurves is 0.22 mag; levels of maxima of the lower lightcurve differ by 0.02 and that of the upper lightcurve by 0 m 05 . In Fig. 1 the positions of the maxima of these lightcurves are shifted from each other. 


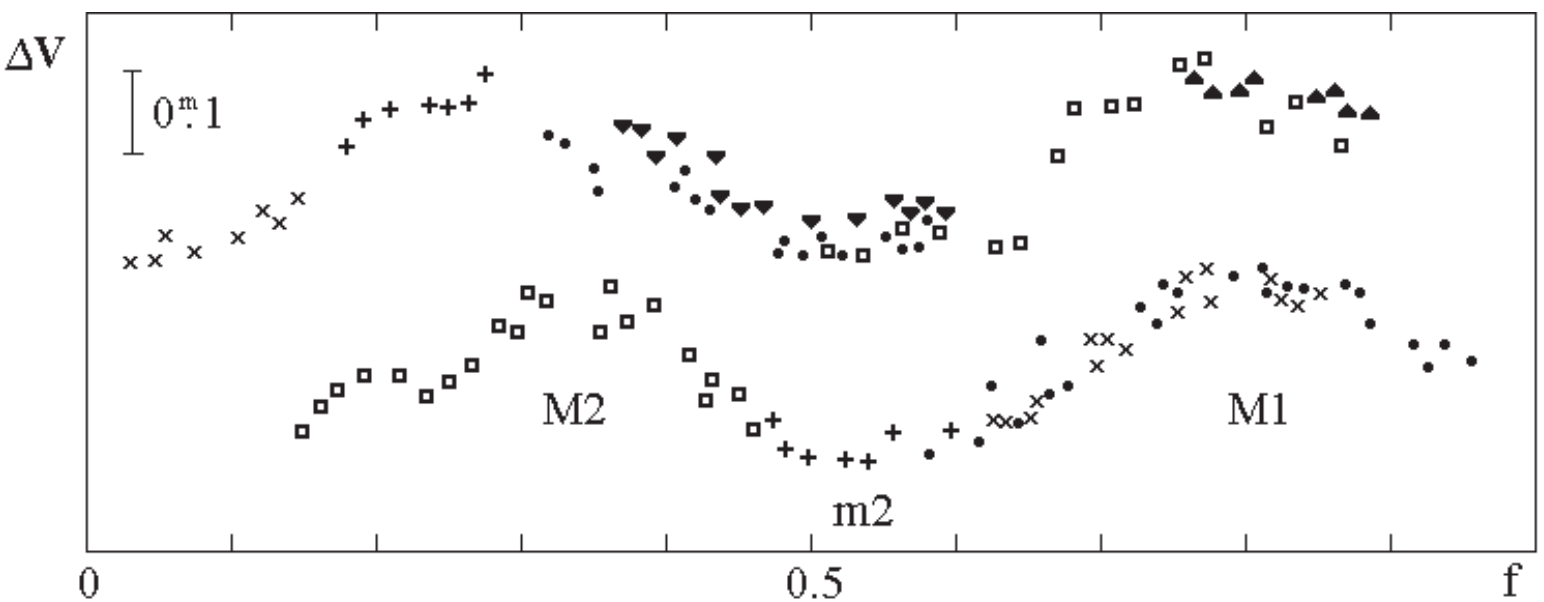

Fig. 1. Composite lightcurves of 44 Nysa: upper - for the interval of phase angles from 0.9 to $3.5\left(0.9-\bullet, 1.2-\square, 1.3-\mathbf{\Lambda}, 2^{\circ} .2-\mathbf{+}, 2^{\circ} .6-\mathbf{\nabla}\right.$, $3.5-\mathbf{x})$; lower - from 5.8 to $8.3(5.8-\boldsymbol{+}, 6.6-\bullet, 7.5-\square, 8.3-\boldsymbol{*})$.

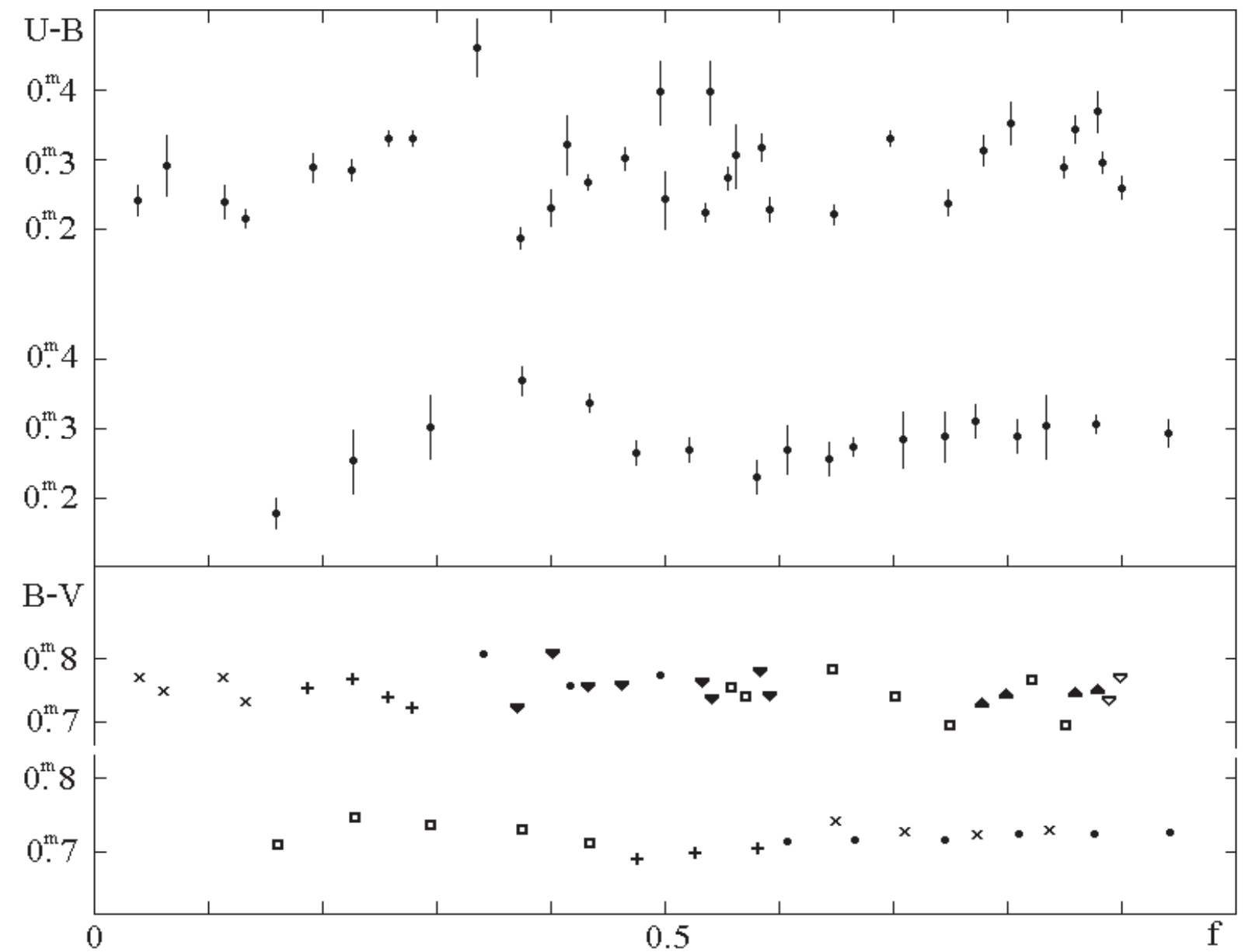

Fig. 2. Composite color curves of 44 Nysa. For $B-V$ on the upper color curve: $\bullet-0.9, \square-1.2, \boldsymbol{\Lambda}-1^{\circ} .3, \mathbf{+}-2^{\circ} .2, \mathbf{\nabla}-2^{\circ} .6, \nabla-3^{\circ} .1, \boldsymbol{x}-3^{\circ} .5$; on the lower color curve: $\mathbf{\uparrow}-5.8, \bullet-6.6, \square-7.5, \boldsymbol{x}-8.3$. The $U-B$ data from each different night are not distinguished by different plot symbols. Phases of rotation of $U-B$ data correspond to the ones of $B-V$ data.

The composite $U-B$ and $B-V$ curves are given in Fig. 2. They were received as lightcurves of Fig. 1, but only for the mean values of color indices in $U-B$ and $B-V$. The mean values of the color indices were calculated as four-point or twopoint floating averages of individual color indices from Table 3. In Fig. 2, vertical lines show the errors of the $U-B$ mean values. The errors of the $B-V$ mean values do not exceed $0{ }^{\mathrm{m}} 01$.
Figure 2 shows the considerable color change over the surface of Nysa in $U-B$ and the faint change in $B-V$. For the interval phase angles from 0.9 to 3.5 the $U-B$ change (the upper curve in Fig. 2) is different to that for the interval $\alpha$ from 5.8 to 8.3 (the lower curve).

The $U-B$ change is seen for the long interval of rotation phase, including the regions of the secondary maximum and 
Table 4. The photometric data for 44 Nysa in 1982.

\begin{tabular}{cccccccccc}
\hline \hline$\alpha$ & $V(\mathrm{M} 1)$ & $V(0.25)$ & $\overline{B-V}$ & $\overline{U-B}$ & $U-B(\mathrm{M} 1)$ & $U-B(0.25)$ & $f$ & Star \\
deg & mag & mag & mag & mag & mag & mag & & \\
\hline 0.9 & 6.97 & 7.04 & 0.74 & 0.37 & 0.35 & 0.34 & $0.33-0.58$ & 2 \\
1.2 & 7.00 & 7.08 & 0.76 & 0.28 & 0.37 & 0.35 & $0.52-0.86$ & 2 \\
1.3 & 7.00 & 7.06 & 0.76 & 0.35 & 0.36 & 0.33 & $0.78-0.88$ & 2 \\
2.2 & 7.04 & 7.10 & 0.74 & 0.30 & 0.34 & 0.33 & $0.19-0.27$ & 3 \\
2.6 & 7.05 & 7.12 & 0.76 & 0.25 & 0.33 & 0.30 & $0.37-0.59$ & 3 \\
3.1 & 7.07 & 7.13 & 0.77 & 0.22 & 0.32 & 0.30 & $0.88-0.90$ & 1 \\
3.5 & 7.08 & 7.14 & 0.75 & 0.21 & 0.32 & 0.30 & $0.04-0.14$ & 3 \\
5.8 & 7.13 & 7.25 & 0.73 & 0.28 & 0.32 & 0.28 & $0.46-0.60$ & 4 \\
6.6 & 7.15 & 7.27 & 0.76 & 0.31 & 0.30 & 0.26 & $0.59-0.95$ & 4 \\
7.5 & 7.18 & 7.31 & 0.74 & 0.31 & 0.30 & 0.26 & $0.14-0.45$ & 4 \\
8.3 & 7.19 & 7.32 & 0.75 & 0.29 & 0.30 & 0.25 & $0.63-0.85$ & 4 \\
11.6 & 7.28 & 7.38 & 0.74 & 0.25 & 0.31 & 0.21 & $0.24-0.34$ & 5 \\
11.8 & 7.29 & 7.36 & 0.75 & 0.23 & 0.31 & 0.21 & $0.80-0.83$ & 5 \\
13.4 & 7.33 & 7.38 & 0.73 & 0.34 & 0.30 & 0.20 & $0.50-0.80$ & 5 \\
15.6 & 7.38 & 7.44 & 0.75 & 0.21 & 0.28 & 0.17 & $0.54-0.64$ & 5 \\
\hline
\end{tabular}

both minima of the lightcurve. The amplitude of the change in $U-B$ is 0.2 . At the region of the secondary maximum of the lightcurve (M2), Nysa is more red than at M1, and at the minima Nysa is more blue.

\section{Phase functions}

In 1982, observations of the asteroid 44 Nysa covered the interval of the solar phase angles from 0.9 to $15^{\circ} 6$ and the aspect angle (the angle between the rotation axes and the line of sight) was changing slightly, not more than $1^{\circ}$. The photometric data for 44 Nysa are given in Table 4, where $V(\mathrm{M} 1)$ and $V(0.25)$ denote the asteroid's brightness reduced to the primary maximum and to the rotation phase $f=0.25$ of the lightcurves in Fig. 1 . These magnitudes were corrected for unit distances $r$ and $\Delta$. $\overline{U-B}$ and $\overline{U-V}$ are the values of mean color indices during the night. $U-B(\mathrm{M} 1)$ and $U-B(0.25)$ are the reduced color $V(\mathrm{M} 1)$ and $V(0.25)$ values.

The phase functions of brightness and color were determined by the data of Table 4 . These functions are given in Fig. 3. Figures $3 \mathrm{a}, \mathrm{b}$ show the reduced brightness $V(\mathrm{M} 1)$ and $V(0.25)$ as a function of the phase angle $(\alpha)$. Figures $3 \mathrm{c}, \mathrm{d}$ show the average colors for each night $\overline{B-V}$ and $\overline{U-B}$ as a function of $\alpha$. In Figs. 3e, g are given the phase functions of the reduced color $U-B(\mathrm{M} 1)$ and $U-B(0.25)$.

It is seen from Figs. $3 \mathrm{a}, \mathrm{b}$ that both functions show a nonlinear increase of the brightness of the asteroid at small phase angles, i.e. an opposition effect. This effect is larger and wider for the phase function which was obtained for $V(0.25)$. Near phase $f=0.25$ Nysa is more blue and less bright than it is at the primary maximum (see Figs. 1 and 2). For $\alpha>5.8$ the phase coefficient $(\beta)$, which determines the linear change of the brightness of the asteroid with phase angle, was calculated for this functions and it is $0.026 \mathrm{mag} / \mathrm{deg}$ for $V(\mathrm{M} 1)$ values and $0.018 \mathrm{mag} / \mathrm{deg}$ for $V(0.25)$.
The mean color indices $\overline{B-V}$ do not change with phase angle (Fig. 3c), while $\overline{U-B}$ decrease with the increase of $\alpha$ (Fig. 3d). The scatter of the points in Fig. 3d was caused by the change of the color $U-B$ with the rotation phase (Fig. 2). After color $U-B$ was reduced to the certain rotation phase this scatter of the points was removed (Figs. 3e, g). In Figs. 3e, g the non-linear change of the $U-B$ color is present for $\alpha<3$ and the linear decrease of the color with the increase in phase angle is present for $\alpha>3^{\circ}$. For the $U-B(\mathrm{M} 1)$ values phase coefficient is $0.002 \mathrm{mag} / \mathrm{deg}$ (Fig. 3e) and $0.011 \mathrm{mag} / \mathrm{deg}$ for the $U-B(0.25)$ (Fig. 3g).

\section{Discussion}

The obtained $U-B$ color change with rotation covers almost $3 / 4$ of the surface of Nysa and has the value of $0.2 \mathrm{mag}$. Nysa is more blue at the minima of its lightcurve than at the secondary maximum (M2), and it is bluer at the primary minimum (m1) than at the secondary minimum $(\mathrm{m} 2)$. The secondary maximum (M2) is slightly redder than the primary maximum (M1). The extended color variation over the surface of Nysa can be caused by the difference in composition or by the difference in surface texture.

In 1977, Nysa was investigated polarimetrically, in order to find an albedo change with rotation (Degewij et al. 1979), but the result was negative. Unfortunately, in 1977 observations were done in other ways than in 1982. In 1977, Nysa's south surface $\left(32^{\circ}\right.$ to the south of the equator) and in 1982 its northern surface $\left(30^{\circ}\right.$ to the north of the equator) were observed.

Note that all other observations of Nysa were done either on the equatorial or the south surface. Only in two oppositions (1982 and 1986) the aspect of the observation was $60^{\circ}$. Pole coordinates of Nysa are taken from Magnussion (1986). Aspect data and color indices for 44 Nysa are summarized in Table 5. 


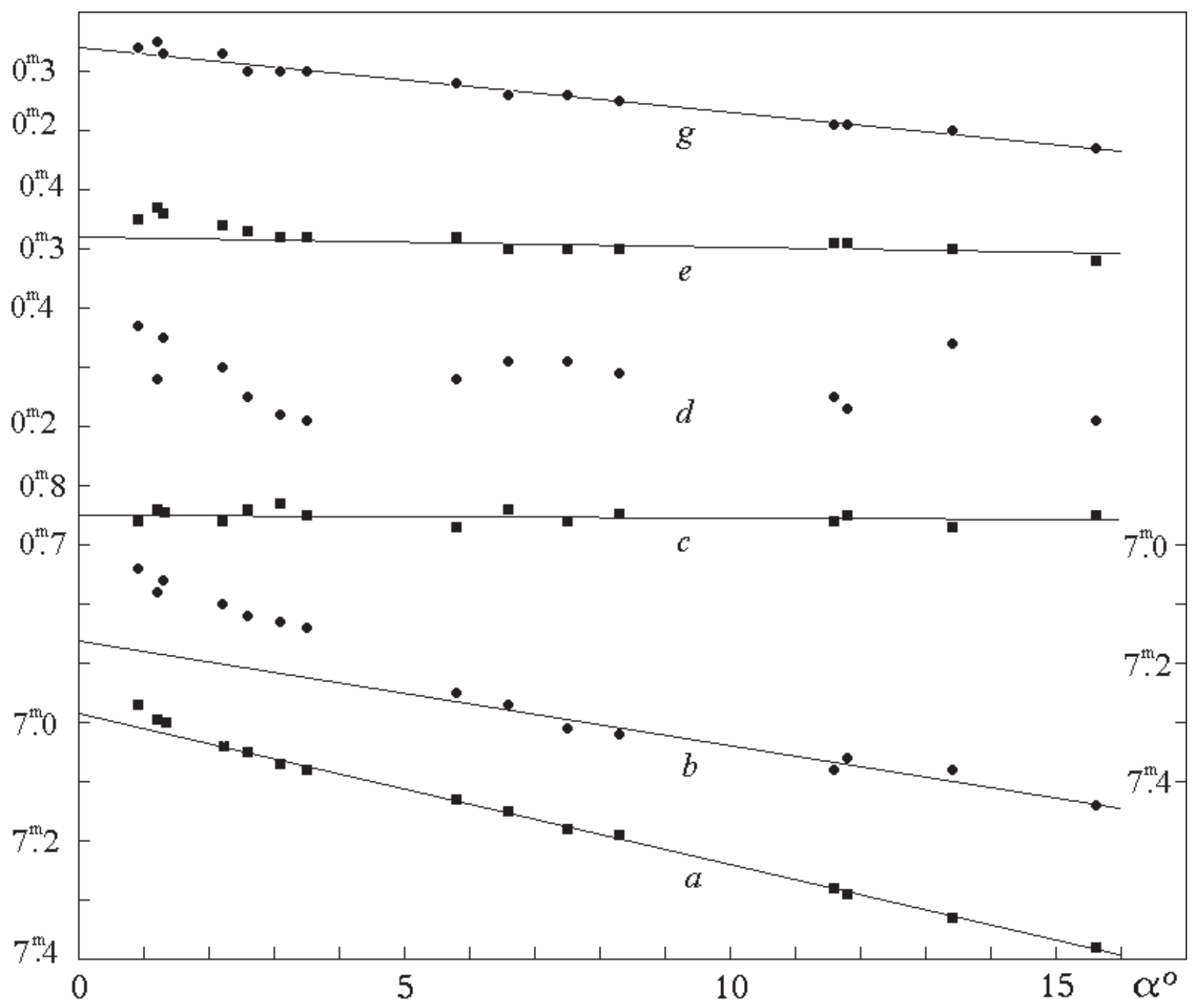

Fig. 3. Phase functions of brightness and colors of Nysa.

Table 5. Aspect and color index of 44 Nysa.

\begin{tabular}{rrrccl}
\hline \hline Year & $\begin{array}{r}\text { Aspect } \\
\text { deg }\end{array}$ & $\begin{array}{r}\alpha \\
\text { deg }\end{array}$ & $\begin{array}{c}U-B \\
\text { mag }\end{array}$ & $\begin{array}{r}B-V \\
\text { mag }\end{array}$ & Ref. $^{a}$ \\
\hline 1954 & -75 & 23 & 0.24 & 0.69 & GK \\
1970 & 86 & 17 & 0.26 & 0.70 & TT \\
1974 & 70 & 5 & - & 0.71 & ZH \\
1975 & 95 & 21 & 0.27 & 0.70 & Z \\
1981 & -74 & 6 & 0.27 & 0.70 & P \\
1982 & 59 & 0.9 & 0.37 & 0.74 & this paper \\
1982 & 59 & 15.6 & 0.21 & 0.75 & this paper \\
\hline
\end{tabular}

a Groeneveld Kuiper (1954) (GK); Piironen (1982) (P); Taylor Tedesco (1983) (TT); Zappala van Houten-Groeneveld (1979) (ZH); Zellner et al. (1977) (Z).

Color indices obtained up to 1982 are smaller than our results. Excluding the possible systematic error of our color indexes, this difference can be explained by the different color of Nysa's two hemispheres. This is possible if Nysa originated from a differentiated parent body, and if there are regions containing different matter on its surface. For example, this effect was observed for the asteroid 15 Eunomia (Reed et al. 1997), which is member of the Eunomia family, like 44 Nysa.

Further interesting results are connected to the phase functions of the brightness and color, which were obtained for different sides of Nysa, namely for the region of the primary maximum (M1) and for the opposite region, which corresponds to the rotation phase 0.25 . Phase functions of the brightness (Figs. 3a, b) have different phase coefficients $(\beta)$ and different values of the opposition effect. Possibly, this is connected to the extended color spot on Nysa's surface. Most of the asteroids for which phase color functions were obtained showed a reddening with phase. Nysa showed an unusual decrease of the $U-B$ color with phase, which differs for the two regions.

Mishchenko \& Dlugach (1993) interpreted Nysa's opposition effect in the $V$ band (Harris et al. 1989) by the coherent backscattering of the light. If coherent backscattering of the light dominates the reflections, then the value of the opposition effect is expected to increase as reflectance increases 
(Nelson et al. 1998). Nysa shows a strong linear decrease of $U-B$ with increase of the phase angle $\left(\beta_{U-B}=0.011 \mathrm{mag} / \mathrm{deg}\right)$ for the surface where the color spot is present $(f=0.25)$ and the observed value of the opposition effect in the $U$ band is smaller than that in $B$ and $V$ bands. Thus, my results confirm that coherent backscattering of the light is the cause of the opposition effect of Nysa.

The opposite side of Nysa $(f=0.8)$ gives a nonlinear change of $U-B$ for small phase angles and a slight linear decrease for $\alpha>5.8$. The opposition effect was not observed in the $U$ band.

\section{Summary}

The color curves connected with the rotation and phase angle were obtained from observations of Nysa in 1982. The color curves show the $U-B$ change with rotation and the value of this change was $0.2 \mathrm{mag}$. These color variations may be connected to the extended color spot on the surface of 44 Nysa.

The $U-B$ color index decreases with the increase in phase angle. A strange $U-B$ decrease is observed for the surface with the color spot.

Phase functions of the brightness, which were obtained for opposite sides of Nysa, differ from each other.

The value of the opposition effect of 44 Nysa decreases as reflectance decreases.
Acknowledgements. I thank Dr. A. W. Harris for the critical comments and suggestions which improved the paper and Dr. P. B. Babadzhanov for assistance and many helpful discussions.

\section{References}

Birch, P. V., Tedesco, E. F., Taylor, R. C., et al. 1983, Icarus, 54, 1 Degewij, J., Tedesco, E. F., \& Zellner, B. 1979, Icarus, 40, 364

Di Martino, M., Zappala, V., De Sanctis, G., \& Cacciatori, S. 1987, Icarus, 69, 338

Groeneveld, I., \& Kuiper, G. P. 1954, ApJ, 120, 529

Harris, A. W., Young, J. W., Contreiras, L., et al. 1989a, Icarus, 81, 365

Harris, A. W., Young, J. W., Bowell, E., et al. 1989b, Icarus, 77, 171 Helfenstein, P., Veverka, J., \& Hillier J. 1997, Icarus, 128, 2

Kazanasmas, M. S., Zavershneva, L. A., \& Tomak, L. F. 1981, Atlas i katalog zvezdnich velichin fotoelectricheskich standartov (Kiev) Magnussion, P. 1986, Icarus, 68, 1

Mishchenko, M. I., \& Dlugach, J. M. 1993, Planet. Space Sci., 41, 173

Moffett, T. J., \& Barnes, T. G. 1979, AJ, 84, 627

Nelson, R. M., Hapke, B. W., Smythe, W. D., \& Horn, L. J. 1998, Icarus, 131, 223

Piironen, J. O. 1982, A\&A, 112, 172

Reed, K. L., Gaffey, M. S., \& Lebofsky, L. A. 1997, Icarus, 125, 446

Taylor, R. C., \& Tedesco, E. F. 1983, Icarus, 54, 13

Vehrenberg, H. 1970, Atlas stellarum 1950.0, Northen part. (Dusseldorf: Trengesell - Verlag)

Zappala, V., \& van Houten-Groeneveld, I. 1979, Icarus, 40, 289

Zellner, B., Andersson, L., \& Gradie, J. 1977, Icarus, 31, 447 